\title{
Science Goals for an Antarctic Large Infrared Telescope
}

\author{
M.G. Burton, J.W.V. Storey and M.C.B. Ashley \\ Joint Australian Centre for Astrophysical Research in Antarctica, \\ School of Physics, University of New South Wales, Sydney NSW 2052, Australia
}

\begin{abstract}
Over the past few years, site-testing at the South Pole has revealed conditions that are uniquely favorable for infrared astronomy. In particular, the exceptionally low sky brightness throughout the near- and mid-infrared leads to the possibility of a modest-sized telescope achieving comparable sensitivity to that of existing 8-10 metre class telescopes. An $8 \mathrm{~m}$ Antarctic telescope, if constructed, would yield performance that would be unrivaled until the advent of the NGST. In this paper we review the scientific potential of infrared telescopes in Antarctica, and discuss their complementarity with existing $8-10 \mathrm{~m}$ class telescopes.
\end{abstract}

Keywords: Antarctica, Site Testing, Astronomy, Infrared, Star Formation, Interstellar Medium, Disks

\section{INTRODUCTION}

The Antarctic plateau provides unique conditions on the Earth for the conduct of observational astronomy. The air is thin, dry and cold and the weather stable; attributes all offering significant sensitivity gains over temperate latitude sites. These conditions are quite different to those experienced at Antarctic coastal locations, which are frequently subject to violent storms.

The plateau is over $3,000 \mathrm{~m}$ in elevation, rising up to $4,300 \mathrm{~m}$ at Dome A. An average year-round temperature of $-50^{\circ} \mathrm{C}$, falling to $-90^{\circ} \mathrm{C}$ at times, vastly reduces the thermal background in the near-IR. A reduced particulate content of the atmosphere lowers the emissivity of the atmosphere in the mid-IR, reducing backgrounds still further. The precipitable water vapour content of the air is typically around $250 \mu \mathrm{m}$ and can fall below $100 \mu \mathrm{m}$, opening up new windows in the infrared and sub-millimeter regimes to ground-based observation. The lack of diurnal temperature variations and the low wind speeds on the highest parts of the Antarctic plateau provide conditions of extraordinary stability, benefiting a wide range of observational programs.

Taken together these conditions provide for an unsurpassed observing environment for Earth-based astronomers across wide ranges of the electromagnetic spectrum, enabling science programs that could only be tackled elsewhere with significantly more expensive facilities. In particular, it will greatly facilitate the pursuit of "formation studies", through new observations in the infrared to millimeter spectral range. These include the study of events such as the formation of galaxies, the birth of the first stars in them and their subsequent evolution, the life cycle of the interstellar medium and the formation of individual stars and planets in our Galaxy. There are three primary reasons why this is so: the continuum emission from these events peaks in the IR, the dominant cooling lines occur across this spectral range, and the cosmic microwave background peaks in the millimeter.

In the remainder of this article we outline the results of the site-testing programs that have quantified the site conditions at the South Pole, and then discuss five science programs which could exploit this unique environment: studies of the large scale environment of star forming complexes, complete determinations of the embedded stellar population within star forming regions, surveys for proto-galaxies, searches for gravitational lensing from stars and planetary systems towards the galactic centre, and interferometric imaging of proto-planetary clouds and Jovian planets towards nearby stars.

Other author information: (Send correspondence to $\mathrm{mgb}$ )

E-mails: M.Burton@unsw.edu.au, J.Storey@unsw.edu.au, M.Ashley@unsw.edu.au 


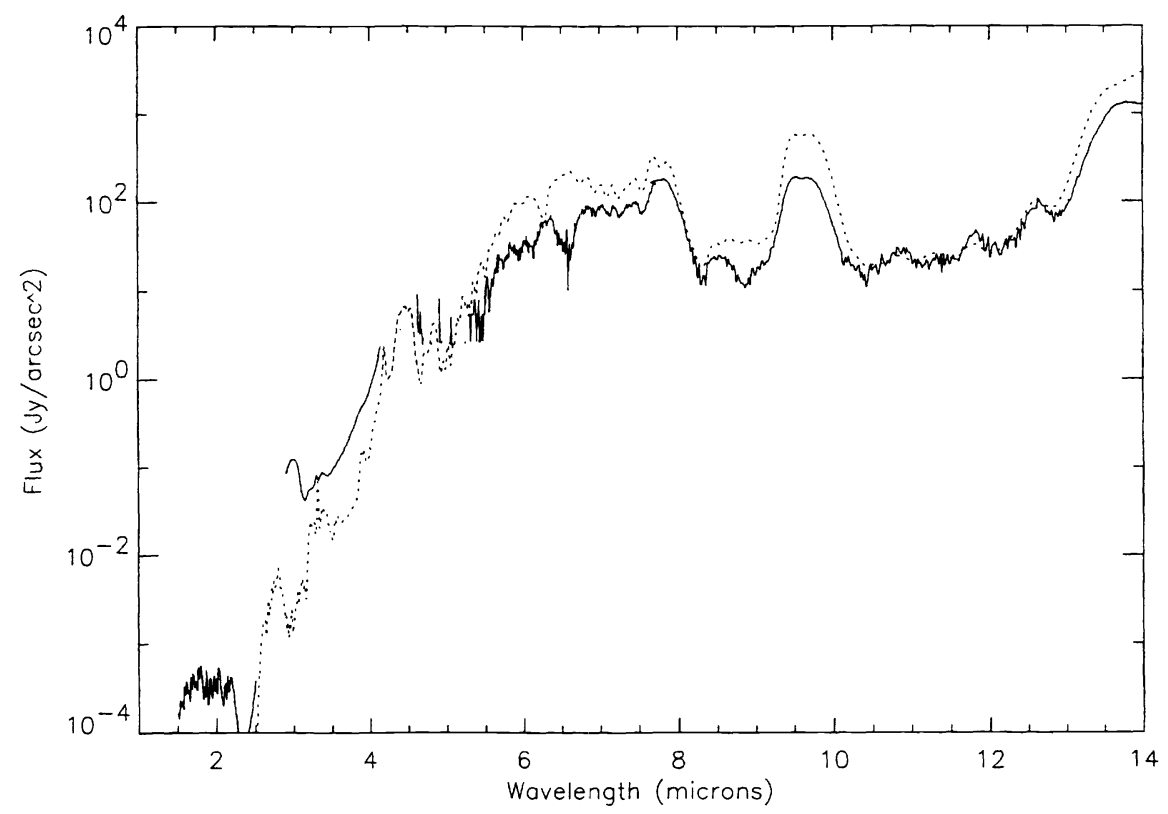

Figure 1. The measured near-IR sky spectrum ${ }^{3}$ (from $1.5-2.5 \mu \mathrm{m}$ and $2.9-4.1 \mu \mathrm{m}$ ) and mid-IR sky spectrum ${ }^{4}$ $(5-14 \mu \mathrm{m})$ at the South Pole. Over-plotted, with a dashed line, is a model spectrum, corresponding to $164 \mu \mathrm{m}$ of precipitable $\mathrm{H}_{2} \mathrm{O}$, plus an aerosol visibility of $100 \mathrm{~km}$. The model fails below $2.3 \mu \mathrm{m}$ because of the neglect of airglow emission.

\section{RESULTS FROM SITE TESTING}

From the extensive site testing program undertaken at the South Pole the following characteristics regarding the infrared background have been determined:

- Sky background in $\mathrm{K}$-dark window $(2.27-2.45 \mu \mathrm{m})$ as low as $\sim 100 \mu \mathrm{Jy} \operatorname{arcsec}^{-2}(20-100$ times less than at temperate sites)..$^{1,2}$

- Sky background in L-band $(3-3.8 \mu \mathrm{m}) \sim 100 \mathrm{mJy} \operatorname{arcsec}^{-2}(\sim 20$ times less than at temperate sites $){ }^{3}$

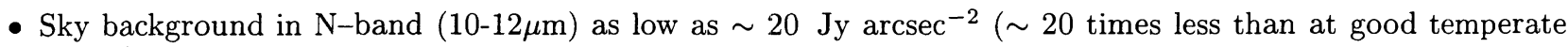
sites). ${ }^{4}$

For background limited imaging of extended regions an Antarctic $2 \mathrm{~m}$ telescope has the same sensitivity as an $8 \mathrm{~m}$ temperate latitude telescope when the background is 16 times lower. It has improved sensitivity for larger background reductions, or if the atmospheric transmission is superior.

In Fig. 1 are shown measured sky spectra from the South Pole, from 2 to $14 \mu$ m, overlaid with a model fit using the atmospheric modelling program MODTRAN. From the parameters to the fit a typical value of $164 \mu \mathrm{m}$ for the precipitable water vapour, with an aerosol visibility of $100 \mathrm{~km}$, was obtained. ${ }^{5}$ This has permitted estimates to be made of the sky background and transparency for wavelengths not covered in the site testing programs. New windows for ground based astronomy are opened in the mid-IR between 20 and $50 \mu \mathrm{m}$, and even windows at 200 and $220 \mu \mathrm{m}$ may be accessible. 


\section{SCIENCE PROGRAMS FOR AN ANTARCTIC INFRARED TELESCOPE}

Having established that the Antarctic plateau provides the best conditions for infrared astronomy on the Earth, it is necessary to consider the demands of the site before determining what programs might best be pursued from there. Clearly, constructing $8 \mathrm{~m}$ size telescopes in Antarctica is a formidable challenge (though much easier than facing the same challenge in space), and the development of Antarctic astronomy will be through smaller facilities first. These will establish the necessary infrastructure, as well as provide test-beds for developing the appropriate engineering technologies and operating practice, that need to be in place before large-scale facilities can be built. Indeed, through the operation of the SPIREX $60 \mathrm{~cm}$ telescope ${ }^{6}$ with the Abu infrared camera, ${ }^{7}$ a prototype system has already demonstrated that infrared astronomy can be conducted from the South Pole over the Antarctic winter.

In view of this necessary staged development program, consideration of appropriate intermediate scale infrared facilities to construct must pay regard to providing complementary functionality to the large $(8 \mathrm{~m})$ telescopes elsewhere, and to exploiting areas where the Antarctic facility clearly provides the most powerful tool for the job. There are three particular niches where an intermediate size ( $2 \mathrm{~m}$ class) Antarctic telescope can be superior to any other telescope on Earth:

- Wide-field thermal infrared imaging. The reduced sky background and improved transmission allow a $2 \mathrm{~m}$ size Antarctic facility to be at least as sensitive as a temperate $8 \mathrm{~m}$ class facility for imaging. Moreover, the Antarctic telescope can survey large areas of sky rapidly in view of its smaller aperture. The telescope, and the instrumentation, is of course vastly cheaper too. Such a facility can complement the $8 \mathrm{~m}$ telescopes by finding the sources for the larger facility to study in depth with high resolution spectrometers.

- Continuous observation at $2.4 \mu \mathrm{m}$, where the sky background is lowest, on sources which are always above the horizon.

- Mid-IR interferometric imaging, exploiting both the greatly reduced background in the $8-12 \mu \mathrm{m}$ range, and the improved sky stability. Such an interferometer may also provide a test-bed for the ambitious space-based projects which aim to detect Earth-like planets around other stars.

We see at least five science programs where these niches will enable significant advances to be made in our knowledge of the Universe:

- Near-IR studies of the environment of embedded star forming complexes, imaging the molecular, neutral and ionized gas through their infrared spectral features.

- Near- and mid-IR imaging of the embedded population of star forming regions, determining their complete population and in particular identifying the youngest members, and the incidence of disks around them.

- Near-IR surveys for proto-galaxies and the early stages of star formation in galaxy evolution.

- Micro-lensing studies of the stars towards the Galactic center at $2.4 \mu \mathrm{m}$, utilising the low sky background and high surface density of stars, in particular to identify the incidence of secondary lensing from planetary systems.

- Mid-IR interferometric imaging of nearby star systems to search for proto-planetary disks, zodiacal dust clouds and Jovian-size planets around them.

We discuss these programs in more detail below.

\subsection{The Environment of Star Forming Complexes}

While massive star formation is one of the most spectacular events in the Galaxy, paradoxically it is poorly understood, because of both the short timescales for the various stages of the process, and because of the many interacting phenomena for which it is hard to disentangle cause and effect. The environment of such star forming complexes, which dominate the southern Galactic plane, can be studied in the thermal infrared through the spectral features from ionized, neutral and molecular species that are present. Embedded HII and UCHII regions can be traced in the $\operatorname{Br} \alpha 4.05 \mu \mathrm{m}$ line. Polycyclic Aromatic Hydrocarbons (PAHs), organic molecules that are fluoresced by far-UV radiation from the young stars and trace the edge of photodissociation regions, are visible through a spectral 


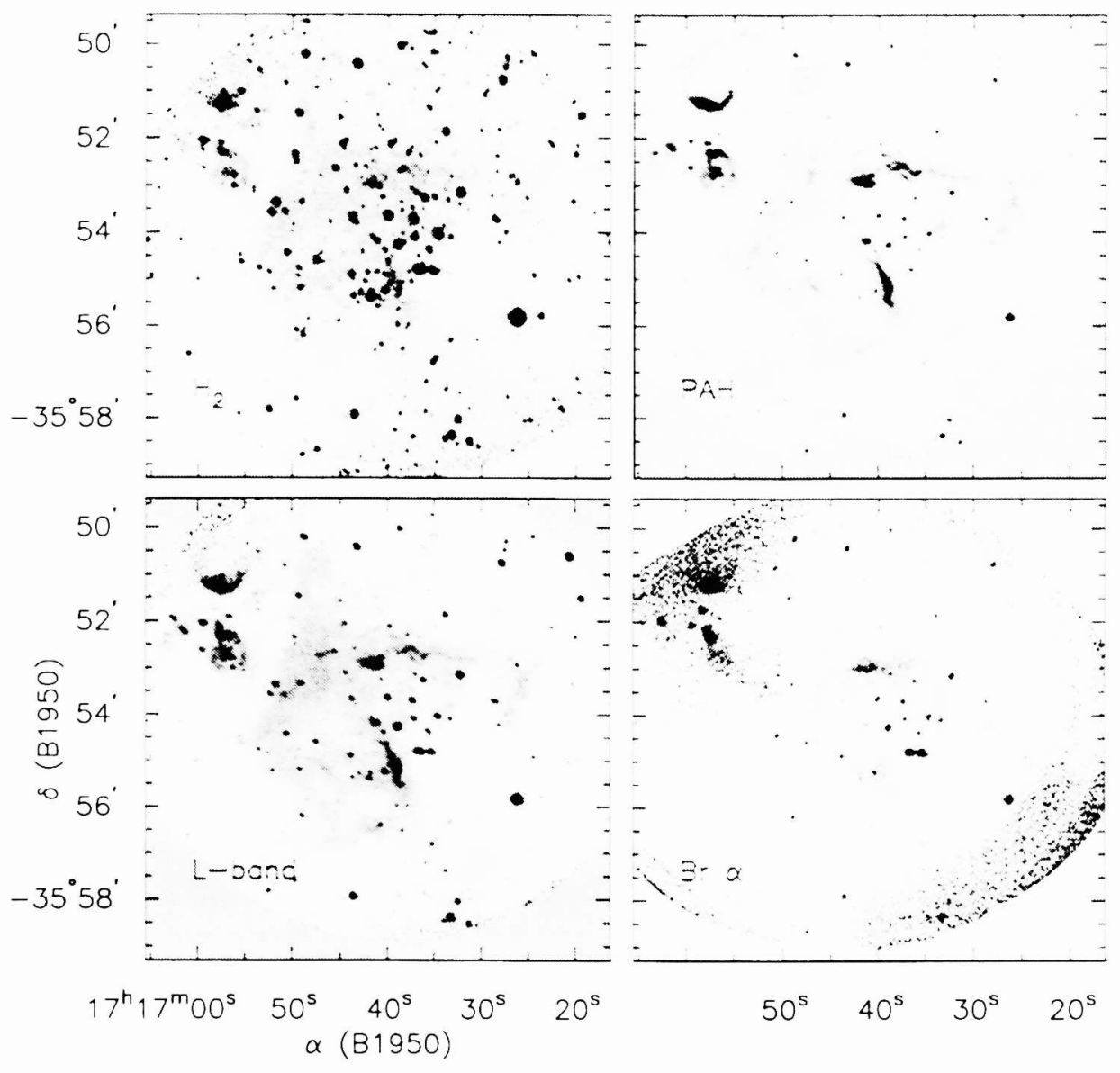

Figure 2. Images ${ }^{8}$ of the SW quadrant of the molecular ridge of NGC 6334, centered on the far-IR source $6334 \mathrm{~V}$. Clockwise, from top left: $\mathrm{H}_{2} 1-0 \mathrm{Q}$ branch $2.42 \mu \mathrm{m}$. $\mathrm{PAH} 3.3 \mu \mathrm{m}, \mathrm{Br} \alpha 4.05 \mu \mathrm{m}$ and $\mathrm{L}$ band $3.5 \mu \mathrm{m}$. 
feature at $3.3 \mu \mathrm{m}$. They can be imaged at high resolution, unlike other prime tracers of these regions, such as the far-IR $\mathrm{C}^{+} 158 \mu \mathrm{m}$ line. Excited $\mathrm{H}_{2}$ emission, resulting from either shocks or UV-fluoresence, can be imaged in the $\mathrm{v}=1-0$ Q-branch lines at $2.4 \mu \mathrm{m}$. Several solid state absorption features are also present, for instance the ice band at $3.1 \mu \mathrm{m}$.

As an example of the potential for this kind of study, Fig. 2 shows a $10^{\prime} \times 10^{\prime}$ region of NGC 6334 , observed with the SPIREX/Abu camera from the South Pole, ${ }^{8}$ in the $\mathrm{H}_{2}, \mathrm{PAH}$ and $\mathrm{Br} \alpha$ features, as well as in the L-band continuum at $3.5 \mu \mathrm{m}$. Shells of photodissociated gas surround bubbles of ionized gas in which embedded, massive protostars reside. Despite the modest size of the SPIREX telescope (just $60 \mathrm{~cm}$ ), these are the deepest images yet obtained at these wavalengths at this spatial resolution. The small aperture, however, also made possible the wide field of view of these images.

\subsection{Complete Population Census of Star Forming Regions}

A key goal for studies of star formation is to undertake a complete population census of star forming clouds in order to determine the number and types of stars that form in them, and how this varies between different complexes. To do so requires observations in the thermal infrared $(\lambda>3 \mu \mathrm{m})$. These wavelengths not only penetrate to the depths of cloud cores, but also allow us to distinguish between the embedded population and background stars. In simple terms, young stellar objects are surrounded by warm (few hundred K) disks which emit strongly at $\lambda>3 \mu \mathrm{m}$, and thus are readily distinguished in infrared color-color diagrams $(e . g$. $[1.65 \mu \mathrm{m}-2.2 \mu \mathrm{m}] /[2.2 \mu \mathrm{m}-3.8 \mu \mathrm{m}])$ from reddened stars. Near-IR color-color diagrams $(e . g . \quad[1.25 \mu \mathrm{m}-1.65 \mu \mathrm{m}] /[1.65 \mu \mathrm{m}-2.2 \mu \mathrm{m}])$, while relatively easy to construct because of the better sensitivities available, show only small IR excesses from the disks. These are readily confused with reddening, and also fail to include the most deeply embedded sources.

The problem has been that at $3.8 \mu \mathrm{m}$ sensitivities are typically $4-5$ magnitudes worse than at $2.2 \mu \mathrm{m}$ from most observing sites, thus limiting the work that has been done in this waveband. Needed are deep, wide-field surveys of comparable sensitivity to those conducted at $2.2 \mu \mathrm{m}$ in order to determine the complete stellar membership of a star formation region. Such an opportunity is afforded by an Antarctic telescope through the greatly reduced thermal background at these wavelengths over temperate sites.

Brown dwarfs - cool sub-stellar objects - may also be identified through the deep absorption band at $3.4 \mu \mathrm{m}$, using narrow band filters on and off the band to determine "colors". Even cooler protostellar objects would be detectable in the mid-IR, for instance embedded sources within 'hot molecular cores', 9 suspected of being the first stage in the process of massive star formation. Imaging through narrow band ( $1 \mu \mathrm{m}$ wide) filters at $8.5,10.5$, and $12.5 \mu \mathrm{m}$, where the background is at a minimum in the mid-IR window, will allow determination of spectral colors of these cooler objects, and thus help to place their evolutionary state.

\subsection{Protogalaxies and the First Star Formation}

The star formation history of the Universe is being probed through deep pencil-beam surveys, of which the Hubble Deep Fields are the most prominent examples. At the faint end of the samples the relative number of peculiar or disturbed galaxies rises dramatically, suggesting that processes to do with star formation (e.g. mergers, starbursts) are active in these sources. However, these galaxies also correspond to the most distant in the samples, with the highest redshift, and in the visible the rest frame being imaged is that of the far-UV. Here star formation is not at its most apparent, and dust absorption can be significant. An Antarctic telescope can search extraordinarily deeply in the $2.4 \mu \mathrm{m}$ "cosmological window", to where for instance the $\mathrm{H} \alpha$ line is red-shifted at $\mathrm{z}=3$, and also provide the first high spatial resolution, wide-field surveys at $3.8 \mu \mathrm{m}$ (L-band), where the visible light from $\mathrm{z}=5$ galaxies would be observed. While the magnitude limit of the HDF $(\mathrm{I} \sim 28)$ will remain far greater than what an Antarctic $2 \mathrm{~m}$ telescope will reach at $3.8 \mu \mathrm{m}$, the colors of high $-\mathrm{z}$ galaxies are particularly red (for instance, an $\mathrm{E} / \mathrm{S} 0 \mathrm{galaxy}$ at $\mathrm{z}=1.4$ has an unreddened color of V-L $\sim 10$ ). Such a galaxy would be readily detectable with an Antarctic $2 \mathrm{~m}$ telescope in a few hours of integration. Moreoever, redder and presumably more interesting galaxies, which were not seen in the HDF, would be detectable. While probing the early universe for protogalaxies is touted as a major project for NGST, the amount of sky surveyed will remain small. An Antarctic $2 \mathrm{~m}$ telescope can play an invaluable complementary role in surveying large areas of sky for such facilities, in order to determine the overall population and distribution of proto-galaxies. NGST will then be able to probe in depth the most interesting sources found. 


\subsection{Micro-lensing towards the Galactic Center}

Gravitational micro-lensing occurs if the geodesic from a star to us passes sufficiently close to a massive, foreground object that its path is bent, or lensed, splitting the light into two images. ${ }^{10}$ If there is a planet near one of the images an additional lensing effect can occur. ${ }^{11}$ The amplitude and light curve of such an event depends on the geometry and mass of the planet, but typically will cause a perturbation on the microlensing light curve with a magnitude of a few percent for a few hours. If there is a planet present the probability of detecting a lensing signature from it is reasonably high if the sampling is frequent and the photometric accuracy high. ${ }^{12}$ To maximize the possibility of finding such events a dedicated telescope should continuously image the same region of sky where the stellar density is high. No where is this more so than towards the Galactic center. Furthermore, the Galactic center becomes readily detectable at $2.4 \mu \mathrm{m}$ (extinction precludes observation at much shorter wavelengths), the very waveband where the sky background is lowest in Antarctica. Moreover, the Galactic center is always visible from the South Pole. For instance, a $2 \mathrm{~m}$ telescope equipped with a $1024^{2}$ array with $0.6^{\prime \prime}$ pixels, mosaicing on a $4 \times 4$ grid, could image a $40^{\prime} \times 40^{\prime}$ region roughly every 20 minutes, achieving a sensitivity of $\sim 17.5$ mags at $2.4 \mu \mathrm{m}$. Towards the Galactic center every pixel would contain at least one star! As calculated by Gould, ${ }^{13}$ the optical depth for lensing is unity; i.e. we would always expect to find at least one lensing event underway. Such a facility would be a powerful tool for exploring the incidence of planetary systems through the micro-lensing signature imposed on the light curve.

\subsection{Interferometry of Proto-Stellar Disks and Jovian Planets}

One of the great challenges facing astronomy, and the focus of major national programs such as NASA's Origins program, is the search Earth-like planets. Several grand design projects have been envisaged towards this goal, for instance NASA's Terrestrial Planet Finder and ESA's Darwin. These are space-based nulling interferometers, a suite of telescopes operating in mid-infrared where the contrast between star and planet is least. Such facilities are not likely to be built before the middle of this century, and many major technological issues remain to be addressed first. Several ground-based interferometers are now under construction, such as the VLT, LBT and the Keck, with the lesser goals of imaging circumstellar disks, zodiacal dust and Jovian planets in nearby stellar systems. An Antarctic infrared interferometer (AII) is an obvious next step after a $2 \mathrm{~m}$ class telescope, exploiting the reduced background, the improved sky stability compared to temperate sites, and the constant airmass of sources. We envisage the AII as a suite of $2 \mathrm{~m}$ size telescopes, initially with just two connected interferometrically, but readily expanded for relatively low cost by the addition of more telescopes, to provide an optimal configuration for imaging other solar systems. It would provide the most powerful ground-based instrument for this purpose.

\section{SUMMARY}

The Antarctic plateau provides a unique environment for infrared astronomy. Intermediate scale, relatively low cost, telescopes could undertake many important scientific programs far more efficiently than other larger facilities. Furthemore, they would complement many in-depth studies planned for the $8 \mathrm{~m}$ class ground-based telescopes and, eventually, NGST. In particular, wide-field thermal IR imaging from 2.4-30 $\mu \mathrm{m}$ would enable comprehensive studies of the star formation, both of the embedded population and the environment in which it occurs within our galaxy, and when it first appears in proto-galaxies. An Antarctic infrared interferometer would provide a powerful facility for the study of nearby solar systems, as well as providing a test-bed for future grand-design space-based facilities that are envisaged in the search for another Earth-like planet.

\section{ACKNOWLEDGEMENTS}

JACARA is the Joint Australian Centre for Astrophysical Research in Antarctica. It is operated by the University of New South Wales and the Australian National University. JACARA's Antarctic astronomy program has been developed through close collaboration with CARA, the US Center for Astrophysical Research in Antarctica. We are grateful to our many colleagues within these organisations for their help and support as we have developed the Antarctic astronomy program in Australia. 


\section{REFERENCES}

1. M. Ashley, M. Burton, J. Storey, J. Lloyd, J. Bally, J. Briggs, and D. Harper, "South pole observations of the near-infrared sky brightness," Pub. Ast. Soc. Pac. 108, p. 721, 1996.

2. H. Nguyen, B. Rauscher, S. Severson, M. Hereld, D. Harper, R. Loewenstein, F. Mrozek, and R. Pernic, "The south pole near-infrared sky brightness," Pub. Ast. Soc. Pac. 108, p. 718, 1996.

3. A. Phillips, M. Burton, M. Ashley, J. Storey, J. Lloyd, D. Harper, and J. Bally, "The near-infrared sky emission at the south pole in winter," ApJ 527, p. 1009, 1999.

4. M. Chamberlain, M. Ashley, M. Burton, A. Phillips, and J. Storey, "Mid-infrared observing conditions at the south pole," $A p J$ in press, 2000.

5. M. Hidas, M. Burton, M. Chamberlain, and J. Storey, "Infrared and sub-millimetre observing conditions on the antarctic plateau," Pub. Ast. Soc. Aust. submitted, 2000.

6. M. Hereld, B. Rauscher, D. Harper, and R. Pernic, "Grim - a near infrared grism and spectrometer," in Instrumentation in astronomy VII, Proc. SPIE 1235, p. 43, 1990.

7. A. Fowler, N. Sharp, W. Ball, A. Schinckel, M. Ashley, M. Boccas, J. Storey, D. Depoy, P. Martini, A. Harper, and R. Marks, "Abu/spirex: South pole thermal ir experiment," in Infrared astronomical instrumentation, A. Fowler, ed., Proc. SPIE 3354, p. 1170, 1998.

8. M. Burton, M. Ashley, R. Marks, A. Schinckel, J. Storey, A. Fowler, M. Merrill, N. Sharp, I. Gatley, D. Harper, R. Loewenstein, F. Mrozek, J. Jackson, and K. Kraemer, "High resolution imaging of photodissociation regions in ngc 6334," Ap. J. submitted, 2000.

9. A. Walsh, F. Bertoldi, M. Burton, and T. Nikola, "Mid-infrared observations of methanol masers sites and ultracompact hii regions," MNRAS submitted, 2000.

10. B. Paczynski, "Gravitational microlensing at large optical depth," ApJ 301, p. 503, 1986.

11. A. Gould and A. Loeb, "Discovering planetary systems through gravitational microlenses," ApJ 396, p. 104, 1992.

12. A. Albrow and et al., "The relative lens-source proper motion on macho 98-smc-1," ApJ 512, p. 672, 1999.

13. A. Gould, "K-band microlensing of the inner galaxy," Ap. J. 446, p. L71, 1995. 